\title{
Infective Endocarditis Caused by Streptococcus Mutans Following Tooth Extraction in a Patient with Undiagnosed Mitral Valve Prolapse Previously: A Case Report
}

\author{
Ilknur Erdem ${ }^{*}$, Enes Ardıç ${ }^{1}$, Ritvan Karaali ${ }^{1}$, Seref Alpsoy², Sahin Topuz ${ }^{2}$, M Enes Kardan ${ }^{1}$, Mustafa \\ Dogan $^{1}$, Selami Gurkan ${ }^{3}$ and Aynur Eren Topkaya ${ }^{4}$
}

${ }^{1}$ Department of Infectious Diseases, Namik Kemal University, Tekirdag, Turkey

${ }^{2}$ Department of Cardiology, Namik Kemal University, Tekirdag, Turkey

${ }^{3}$ Department of Cardiovascular Surgery, Namik Kemal University, Tekirdag, Turkey

${ }^{4}$ Department of Medical Microbiology, Namik Kemal University, Tekirdag, Turkey

*Corresponding author: Ilknur Erdem, Faculty of Medicine, Department of Infectious Diseases, Namik Kemal University School of Medicine, 59100, Tekirdag, Turkey, Tel: +90-532-5888235; E-mail: ilknurerdem@hotmail.com

\begin{abstract}
Infective endocarditis is an acute or a subacute inflammatory infection of the endocardium caused by bacterial, viral or fungal microorganisms. Despite significant improvements in the diagnosis and treatment, it is still associated with a poor prognosis and a high mortality rate. Viridans streptococci are one of the most common causative microorganisms of bacterial endocarditis. Streptococcus mutans, a member of this group and is usually associated with dental carries. Although it is expected to be more frequently associated with endocarditis, has only rarely been reported. This is most likely to be due to some identification problems. We herein report a case of infective endocarditis due to Streptococcus mutans following tooth extraction with no history of heart disease.
\end{abstract}

Keywords: Infective endocarditis; Streptococcus mutans; Mitral valve prolapse

Received Date: March 12, 2018; Accepted Date: March 27, 2018; Published Date: March 31, 2018

\section{Introduction}

Infective endocarditis (IE) is defined by infection of a native or prosthetic heart valve, the endocardial surface, or an indwelling cardiac device. Despite advances in medical and surgical therapy, IE remains associated with high mortality rate and serious complications such as heart failure, hearth rhythm problems, stroke, infections in other organs (lungs, brain or kidneys) or even death. Intravenous drug use, recent invasive procedures (dental work), history of previous IE, and congenital or acquired heart lesions are all risk factors for IE. Transient bacteriemia usually occurs during dental, gastrointestinal, and urological procedures and after such interventions; IE incidence is increased in susceptible patients at risk for development. The most frequent IE 
www.tridhascholars.org | April-2018

pathogens are Gram-positive bacteria- S. aureus, Streptococcus spp., and Enterococci are responsible for more than $80 \%$ of all IE cases. Mitral and aortic valve involvement is most frequent site [1-3].

Viridans group streptococci (VGS) are the dominant members of the oral microbiota. Streptoccocus mutans (S. mutans), a member of the VGS, which is also associated with dental carriage, is expected to be more frequently isolated as an endocarditis, but less reported. It is thought that the less isolation of $S$. mutans may be due to some identification problems. $S$. mutans may be mistakenly defined as Enterococci, Diphteroids or Lactobacilli, or S. viridans or Microaerophilic streptococci. The morphology of $S$. mutans is similar to that of Diphtheroids at low $\mathrm{pH}[1,3-6]$.

Mitral valve prolapse (MVP) is a valvular disease characterized by left atrial valve dislocation during systole of one or both mitral valve. It is generally considered to be the most common cardiac valvular disease. Complications such as ventricular extra systole, mitral insufficiency and infective endocarditis may develop rarely in this disease. In patients with MVP, the IE risk is 8 times more than the normal population [7]. A strong association between mitral valve prolapse and VGS IE was reported [8].

Although S. mutans, a major pathogen of dental caries, is rarely reported as the etiological agent in patients with IE due to some identification problems [5,9]. Here we present a case of IE due to S. mutans after tooth extraction. The patient had no known underlying heart disease previously and were diagnosed with MVP.

\section{Case Report}

A 49-year-old male patient was admitted to our clinic with complaints of sub-febrile fever, sweating, weakness, shortness of breath, weight loss for one month. The patient who had not known heart disease had a tooth extraction story without antibiotic prophylaxis a month and a half ago. On the physical examination, the general condition of the patient was moderate, consciousness was clear. Fever was $36.7^{\circ} \mathrm{C}$, pulse was 85 beats/minute, arterial blood pressure was $120 / 70 \mathrm{mmHg}$. A $3 / 6$ pansystolic murmur was heard over the apex. In the tests performed, Hgb was $11.9 \mathrm{gr} / \mathrm{dl}$, Wbc was 11000/mm3, C-reactive protein was $74 \mathrm{mg} / \mathrm{dl}$ and sedimentation rate was 53/h. Transthoracic echocardiography was revealed fibrotic changes on the mitral valve tip and papillary muscles of the tricuspid and aortic valves. In the transesophageal echocardiography, $3 \times 3 \mathrm{~mm}$ vegetation on the mitral valve atrial surface, mitral valve regurgitation, and MVP were reported. Abdominal ultrasound was revealed a decrease parenchymal echogenicity in liver and spleen. Three sets of blood cultures and urine cultures were taken from the patients in the first day of hospital admission. On the fourth day of admission to the hospital, the patient had a fever of $38.5^{\circ} \mathrm{C}$, and 3 sets of blood cultures were taken again. Empirically, Ampicillin-Sulbactam $4 \times 3$ gr IV and gentamycin $1 \times 160$ mg IV treatment started. On the $5^{\text {th }}$ day of the hospitalization, all blood cultures were positive for growth of $S$. mutans, sensitive to Ampicillin. According to the modified Duke criteria [10], IE was diagnosed with 2 major and 2 minor criteria. The patient's treatment was continued with Ampicillin $4 \times 3 \mathrm{gr}$ IV for four weeks and Gentamycin $1 \times 160 \mathrm{mg}$ IV for two weeks. $15^{\text {th }}$ day of the treatment, $3 \times 3 \mathrm{~mm}$ vegetation on the atrial surface of the mitral valve, cord tendinea rupture and severe mitral regurgitation were detected by the control TEE. CVS clinic was consulted for the operation and due to the general condition and vital signs of the patient being stable, operation was planned after the completion of the IE antibiotic treatment. The patient who received four weeks of parenteral treatment completed the IE treatment. Mitral valve replacement operation was performed to the patient after transferred to the CVS clinic.

\section{Discussion}

The incidence of IE is a rare with a rate of 1.7-5/year in 100.000 people. Males are more common than females. In spite of 
www.tridhascholars.org | April-2018

developments in the diagnosis and management of infective endocarditis, this disease still carries high rates of morbidity and mortality. Dental procedures may be associated with transient bacteriemia. The incidence of bacteraemia following dental procedures such as tooth extraction, endodontic treatment, periodontal surgery, and root scaling has been well documented $[1,2,11]$. In our patient, tooth extraction was performed 15 days before complaints began. No prophylaxis was performed before tooth extraction.

VGS are one of the most common causative microorganisms of IE [3]. In a review of 848 cases with IE, the most frequently isolated microorganism was Streptococci spp. (49.5\%) followed by Staphylococci spp. (31.7\%) [11]. The most common streptococci species in patients with IE were Streptococcus sanguinis while $S$. mutans was reported to be responsible for approximately $15 \%$. S. mutans, a pathogen of dental caries, is known to be associated with bacteraemia and infective endocarditis. It is expected to be isolated more frequently as an endocarditis causative agent but probably less reported that may be due to identification problems. Of VGS cultures from gingiva and blood after dental procedures, 19\% were tolerant, especially among S.mutans (27\%) and S.mitior (20\%) isolates. Isolates of S. mutans should be tested for tolerance that would require the addition of an aminoglycoside to the penicillin regimen $[1,2,4,5]$. In one study from Turkey, it was reported the rates of resistance and reduced sensitivity among the S. mutans isolates for Penicillin and Ampicillin were determined at 66\% and $50 \%$, respectively [12]. In our case, S.mutans was sensitive to all tested antimicrobials.

IE caused by VGS is subacute and there is an underlying cardiac disease in the majority of patients. VGS are the most frequently isolated pathogen associated with MVP in IE cases. In patients with MVP, the likelihood of development of IE is higher than in normal populations. Especially, as the degree of mitral regurgitation increases, the amount of mechanical and hemodynamic stress increases, which can increase the endothelial damage and bacterial adhesions with in bacteremia [7,8]. In our patient who had no known heart disease had a tooth extraction story without antibiotic prophylaxis a month and a half ago.

Penicillin G (12-18 $\mu \mathrm{M}$ ), Ampicillin (2 g every 4 hours or $3 \mathrm{~g}$ every 6 hours, IV), or ceftriaxone $2 \mathrm{~g} /$ day for 4 weeks may be given for Penicillin susceptible isolates in the IE treatment of VGS. If combination therapy (Gentamicin and Penicillin) is administered in strains with penicillin susceptibility, the duration of treatment may be reduced from 4 weeks to 2 weeks. Vancomycin (15-20 mg/kg/day, IV 8-12 hour intervals, 6 weeks) and gentamicin (3 mg/kg/day, IV single dose or 2-3 times a day in equal doses) with Penicillin allergy and Penicillin resistant cases. In our case, combination treatment with Ampicillin ( $4 \times 3 \mathrm{~g}$, IV) for 4 weeks and Gentamicin $(1 \times 160 \mathrm{mg}$, IV) for 2 weeks was administered according to the antibiotic susceptibility result that the isolate was a sensitive to Penicillin (Penicillin MIC <0.06) $[1,2,13]$.

In some cases, surgery is necessary to repair or replace a damaged heart valve. According to the recent literature of the American Heart Association, surgical treatment should be planned with medical treatment in cases like a large systemic embolic episode, with large ( $>1 \mathrm{~cm}$ in diameter) anterior mitral valve vegetations, increased vegetation size after appropriate antibiotic treatment, acute mitral insufficiency, ruptured heart valve and perianal dilatation, myocardial abscess $[1,2,13]$. In our case, the operation decision was taken because of the rupture of the cord tendon in the mitral valve at the control TEE performed during the second week of the patient's treatment. After the four weeks of antibiotic therapy, he was transferred to the CVC Clinic. The patient underwent mitral valve replacement and was seen to have improved in his clinic.

\section{Conclusion}


www.tridhascholars.org | April-2018

IE is a serious and life-threating disease. S. mutans, an important cause of dental caries and endocarditis, may be easily misdiagnosed by the inexperienced microbiologist as a common non-pathogenic skin bacterial contaminant termed Diphtheroid. It is important to kept that misidentification of a major pathogen can cause delay in the diagnosis and treatment of a serious infection.

\section{Consent}

The authors declare that informed written consent was obtained from the patient for the publication of this manuscript.

\section{References}

1. Fowler VG, Scheld Jr WM, Bayer AS (2015) Endocarditis and intravascular infections. In: Bennett JE, Dolin R, Blaser MJ (eds). Mandell, Douglas and Bennett's Principles and Practice of Infectious Diseases. Philadephia: Elsevier Saunders, $8^{\text {th }}$ edn. p 990-1019.

2. Moreillon P, Que YA (2004) Infective endocarditis. Lancet 363 (9403): 139-149.

3. Everett ED and Hirschmann JV (1977) Transient bacteremia and endocarditis prophylaxis. A review. Medicine 56(1): $61-77$.

4. McGhie D, Hutchison JG, Nye F, et al. (1977) Infective endocarditis caused by Streptococcus mutans. Heart, 39(4): 456-458.

5. Ullman RF, Miller SJ, Strampfer MJ, et al. (1988) Streptococcus mutans endocarditis: report of three cases and review of the literature. Heart \& Lung: the Journal of Critical Care 17(2): 209-212.

6. Nakano K, Nomura R and Ooshima T (2008) Streptococcus mutans and cardiovascular diseases. Japanese Dental Science Review 44(1): 29-37.

7. Katan O, Michelena HI, Avierinos JF, et al. (2016) March. Incidence and predictors of infective endocarditis in mitral valve prolapse: a population-based study. In Mayo Clinic Proceedings 91(3): 336-342.

8. DeSimone DC, DeSimone CV, Tleyjeh IM, et al. (2015) Association of mitral valve prolapse with infective endocarditis due to viridans group Streptococci. Clinical Infectious Diseases 61(4): 623-625.

9. Schelenz S, Page AJF and Emmerson AM (2005) Streptococcus mutans endocarditis: beware of the 'Diphtheroid'. Journal of the Royal Society of Medicine 98(9): 420-421.

10. Li JS, Sexton DJ, Mick N, et al. (2000) Proposed modifications to the Duke criteria for the diagnosis of infective endocarditis. Clinical Infectious Diseases 30(4): 633-638.

11. Seymour RA, Lowry R, Whitworth JM et al. (2000) Infective endocarditis, dentistry and antibiotic prophylaxis; time for a rethink?. British Dental Journal 189(11): 610.

12. Süzük S, Kaşkatepe B and Çetin M (2016) Antimicrobial susceptibility against penicillin, ampicillin and vancomycin of viridans group Streptococcus in oral microbiota of patients at risk of infective endocarditis. Le Infezioni in Medicina 24(3): 190-193.

13. Nakatani S, Mitsutake K, Hozumi T, et al. (2003) Committee on Guideline for Prevention and Management of Infective Endocarditis, Japanese Circulation Society. Current characteristics of infective endocarditis in Japan: an analysis of 848 cases in 2000 and 2001. Circulation Journal 67: 901-905. 